\title{
Control of the Properties of Micro-Structured Waveguides in Lithium Niobate Crystal
}

\author{
Huseyin Karakuzu, Mykhaylo Dubov, Sonia Boscolo \\ Aston Institute of Photonic Technologies, School of Engineering and Applied Science, Aston University, Birmingham \\ B4 7ET, United Kingdom \\ m.dubov@aston.ac.uk
}

Leonid Melnikov, Yulia Mazhirina

State Technical University, 77 Politekhnicheskaya str., 410054, Saratov, Russia

mazhirina@mail.ru

\begin{abstract}
We study numerically depressed-cladding, buried waveguides that can be formed in a lithium niobate crystal by femtosecond laser writing. We demonstrate that the guiding properties can be controlled by the waveguide structural characteristics

(C) 2013 Optical Society of America

OCIS codes: $130.3730,130.5296,140.3390$
\end{abstract}

\section{Introduction}

The combination of electro-optical, acousto-optical and nonlinear $\left(\chi^{(2)}\right)$ optical properties, as well as its wide transparency window, make lithium niobate $\left(\mathrm{LiNbO}_{3}\right)$ an attractive host material for application in new generation integrated optics. As one of the most efficient techniques for three-dimensional volume microstructuring of transparent dielectrics, the femtosecond (fs) laser inscription method has been widely applied to fabricate optical waveguides (WGs) in numerous optical materials, including optical crystals, ceramics, glasses and polymers [1]. Tight focusing of an intense fs laser beam inside a transparent crystal can be used to write multiple tracks with a reduced refractive index (RI) around the unmodified volume of material, thus producing a depressed-cladding with the central volume serving as the core of a WG [2]. Due to geometric flexibility, the cladding may consist of a fairly large number of arbitrarily arranged tracks confining the flexibly large and shaped guiding area [3]. In this paper, we study numerically depressedcladding, buried WGs that can be formed in a Z-cut $\mathrm{LiNbO}_{3}$ crystal by fs laser writing, in a pursuit of establishing how experimentally accessible parameters, such as the number, position, size and shape of the cladding tracks, and the RI contrast between the low-index cladding structure and the core guiding region, can be used for achieving control over the propagation constants of modes of different polarisations, hence the conditions for quasi-phase-matching in a WG geometry, similarly to Photonic Crystal Fibre (PCF), where tracks with a reduced RI are naturally formed during the drawing process. The results presented can help designing various NIR and mid-IR infrared integrated optical components: phase preserving, low-noise parametric amplifiers with a small footprint based on WGs in $\chi^{(2)}$ materials, synchronously-pumped single-photon (quantum) sources and ultra-fast, single-photon mid-IR detectors realized by WG-based frequency converters, all-optical wavefront generators, optical pulse shapers etc.

\section{Waveguide geometry and methodology}

The depressed cladding, 'leaky-mode' can be formed by a number of concentric, hexagonal lattices of uniformly spaced, equally-sized cylindric tracks (Fig. 1 top), which can be written in a $\mathrm{LiNbO}_{3}$ crystal by direct fs laser irradiation using a transverse inscription geometry [1].

The reduced RI of the tracks was assumed to be uniform across the cross section of a track, and polarisation and wavelength-independent. Key parameters that were varied in the numerical model include the tracks' diameter $D$ and spacing $a$, track ellipticity $e l$, the RI contrast between the cladding and core regions, and the number of tracks' lattices (depressed-cladding layers). We solved wave equation

$$
\nabla \times\left(\hat{\varepsilon}^{-1} \nabla \times \vec{H}\right)=k^{2} \vec{H},
$$

where $k$ is the wave number in vacuum. For an uniaxial crystal hosting a depressed-cladding WG with the RI contrast $\delta n$, the permittivity tensor can be written as $\varepsilon_{i j}=\left(n_{i}+\delta n\right)^{2} \delta_{i j}, i, j,=x, y, z, n_{i}=\left\{n_{o}, n_{e}, n_{o}\right\}$ where $n_{o}, n_{e}$ are the 


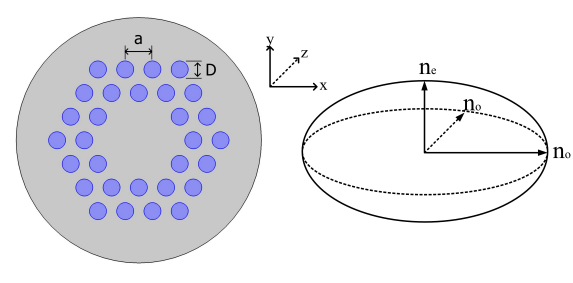

a)

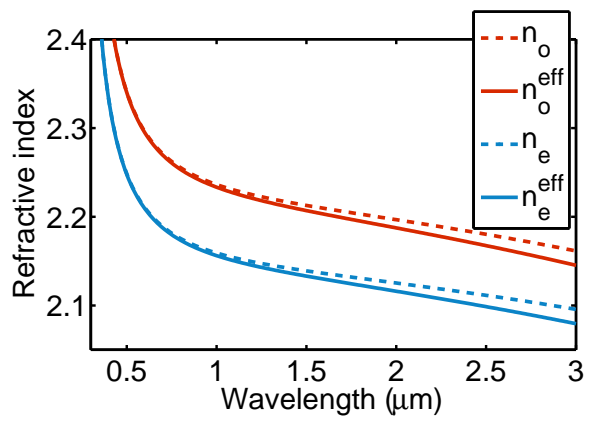

b)

Fig. 1. Cross-section of a 2-layered depressed-cladding WG and index ellipsoid for LiNbO3 host (a). Real parts of effective RIs, $n_{\text {eff }}^{o, e}$ of the WG as a function of wavelength (b). The RIs of the unmodified material $n_{o, e}$ are also shown. WG parameters are: $D=1.6 \mu \mathrm{m}, a=2 \mu \mathrm{m}, \delta n=-0.05$.

ordinary and extraordinary RIs of the intact domain of crystal, $\delta n$ is nonzero only in the irradiated domain, and light is assumed to propagate along the $x$ axis of the crystal. Equation (1) can be solved using the COMSOL simulation software to find out the propagation constants, and effective RIs $n_{e f f}^{o, e}$, of propagation modes polarised along the orthogonal directions $y$ and $z$. The wavelength was varied in the transparency window of $5 \% \mathrm{MgO}$-doped $\mathrm{LiNbO}_{3}$ [3], and the Sellmeier equations were used to compute $n_{o, e}$ [4]. A perfectly matched layer (PML) absorber was used to truncate the computational domain and, thus, minimize the effect of boundary reflections $[5,6]$. For investigation of tracks ellipticity effect and calculation of cladding modes dispersion diagramme the plane-wave-decomposition $[7,8]$ was used. Assuming $x$-dependence of the fields as expi $\beta x$ from (1) equations for for $H_{y}(y, z)$ and $H_{z}(e, z)$ can be derived:

$$
\pm \frac{1}{\eta_{z, y}} \frac{\partial \eta_{x}}{\partial z, y} \frac{\partial H_{y, z}}{\partial z, y} \mp \frac{1}{\eta_{z, y}} \frac{\partial \eta_{x}}{\partial z, y} \frac{\partial H_{z, y}}{\partial y, z}+\frac{\partial^{2} H_{y, z}}{\partial y, z^{2}}+\frac{\eta_{x}}{\eta_{z, y}} \frac{\partial^{2} H_{y, z}}{\partial z, y^{2}}+\left(1-\frac{\eta_{x}}{\eta_{z, y}}\right) \frac{\partial^{2} H_{y, z}}{\partial y \partial z}=\beta^{2} H_{y, z}(y, z),
$$

where $\eta_{i}=\varepsilon_{i i}^{-1}, i=x, y, z$. These equation were solved numerically assuming periodical variations of $\eta_{i}$ and $H_{y, z}$ :

$$
H_{y, z}=\sum_{m, n} H_{y, z}^{m n} \exp \left[i\left(G_{m n}^{y} y+G_{m n}^{z} z\right)\right], \eta_{i}(y, z)=\sum_{m, n} \eta_{i}^{m n} \exp \left[i\left(G_{m n}^{y} y+G_{m n}^{z} z\right)\right] .
$$

Here $G_{m n}^{y, z}$ are determined by unit cell shape in yz-plane. The structure can be specified both analytically (via coordinates of the standard shapes: circles, ellipses, etc.) and even using bitmap photos or drawings.

\section{Results and discussion}

Figure 1 (bottom) shows the wavelength dependencies of the real parts of the ordinary and extraordinary effective RIs of a two-layered depressed-cladding WG. The difference between the group-velocity dispersion profile in the WG and the dispersion in the unmodified material for the ordinarily polarised wave is plotted in Fig. 2a for different values of $\delta n$. While these values are at or above the RI contrasts of low loss depressed WGs that can be achieved with current fs micro-fabrication technology in $5 \% \mathrm{MgO}: \mathrm{LiNbO}_{3}$, the corresponding dispersion changes are quite small. This is because with only two depressed-cladding layers, the mode leakage out of the WG is large. We also observed that increasing either the size $D$ of the tracks or the separation a between tracks led to less dispersion variations. This result was expected since only a compact cladding structure with small-sized and densely packed tracks may eventually form a bandgap which enables both large change in the dispersion and low-loss propagating modes. The case described in Fig. $2 \mathrm{~b}$ also reveals a significant reduction in the propagation loss (imaginary part of the effective RI) for increasing number of layers. The propagation loss for the extraordinary polarisation can be reduced by four orders of magnitude at the wavelengths around $1550 \mathrm{~nm}$ by increasing the number of layers from 2 to 7 . We would like to note, however, that in practice the total propagation losses will always be higher than the values reported here because of various factors, including: material absorption induced by fs irradiation, scattering losses due to irregularities (non-smoothness) of the cladding tracks, and imperfect positioning of the tracks which would increase the mode leakage out of the band-gap. In Fig. 3 two-core and one core structures with elliptical tracks are shown, demonstrating the even and odd modes in 

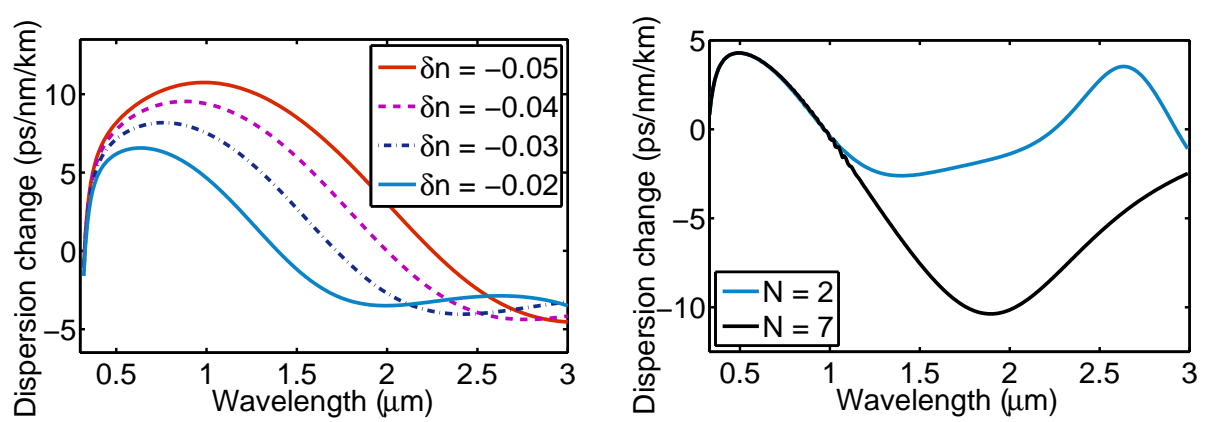

a)

b)

Fig. 2. (a) Difference between dispersion profile in a 2-layered depressed-cladding WG and dispersion in the unmodified $\mathrm{LiNbO}_{3}$ crystal for ordinary polarisation, for varying values of $\delta n$. WG parameters are: $D=1.6 \mu \mathrm{m}, a=2 \mu \mathrm{m}$. (b) The same for varying number $\mathrm{N}$ of depressed-cladding layers, while WG parameters are: $D=1.6 \mu \mathrm{m}, a=2 . \mu \mathrm{m}, \delta n=-0.01$.

extra-ordinary waves. It should be noted that ordinary wave modes have propagation constant close to ones of cladding modes, thus it is expected that these modes are unstable against polarisation disturbance.

\section{Conclusions}

We have demonstrated that the guiding properties of depressed-cladding, buried WGs formed in a $\mathrm{LiNbO}_{3}$ crystal by fs laser writing can be controlled by the WG structural characteristics, even for the relatively moderate induced RI contrasts typical of the direct fs inscription. The number of depressed-cladding layers has revealed to play a major role in the control of the WG properties. We have shown that for an induced RI contrast of -0.01 , the propagation losses can in principle be reduced by four orders of magnitude at telecom wavelengths by increasing the number of cladding layers from 2 to 7.
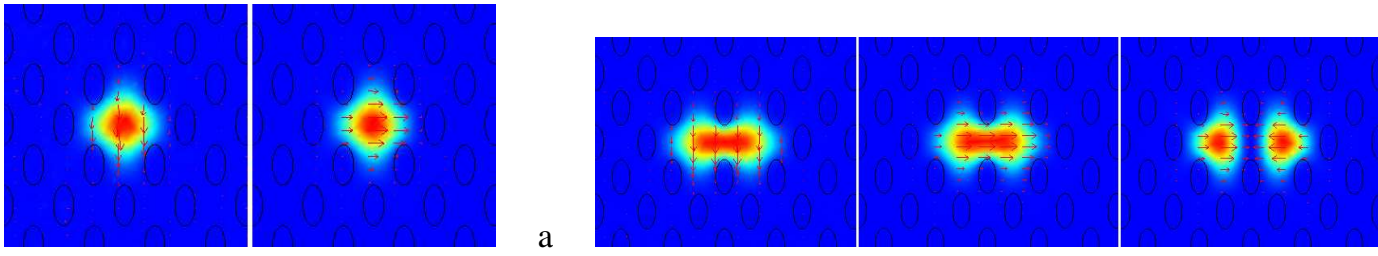

Fig. 3. Fundamental modes fields for one core (a) and two-core (b) structures: $D=1 \times 2 \mu \mathrm{m}, a=3$ $\mu \mathrm{m}, \delta n=-0.05$. Left pictures $(\mathrm{a}, \mathrm{b})$ present field distributions for ordinary polarised modes.

\section{References}

1. R. Osellame et al. eds., Femtosecond laser micromachining, Springer-Verlag (2012).

2. A. M. Streltsov, Proc. SPIE 4941, 51 (2003).

3. A. G. Okhrimchuk et al., Opt. Lett. 30, 2248 (2005).

4. D. E. Zelmon et al., J. Opt. Soc. Am. B 14, 3319 (1997).

5. Y. Tsuji et al., J. Lightwave Technol. 18, 618 (2000).

6. A. Oskooi et al., J. Comput. Phys. 230, 2369 (2011).

7. J. Joannopoulos, et.al., Photonic Crystals: Molding the Flow of Light. Princeton Univ.Press, 2008.

8. Yu. Mazhirina, et al. Opt. Spectr. 107, 454 (2009) 